Supporting Information

\title{
Disposable Polyurethane Nanospiked Gold \\ Electrode-Based Label-Free Electrochemical \\ Immunosensor for Clostridium difficile
}

Feiyun Cui, ${ }^{\dagger}$ Zhiru Zhou, ${ }^{\dagger}$ Hanping Feng, ${ }^{\star}$ H. Susan Zhou, *’’

${ }^{\dagger}$ Department of Chemical Engineering, Worcester Polytechnic Institute, 100 Institute Road,

Worcester, MA 01609, United States

$\$$ Department of Microbial Pathogenesis, University of Maryland Dental School, 650W.

Baltimore Street, Baltimore, MD 21201, United States

Corresponding author

*E-mail address: szhou@wpi.edu (H.S.Z.) 


\section{Characterization of PU Nanospiked Gold Electrode by Energy Dispersive X-ray Spectroscopy (EDS)}

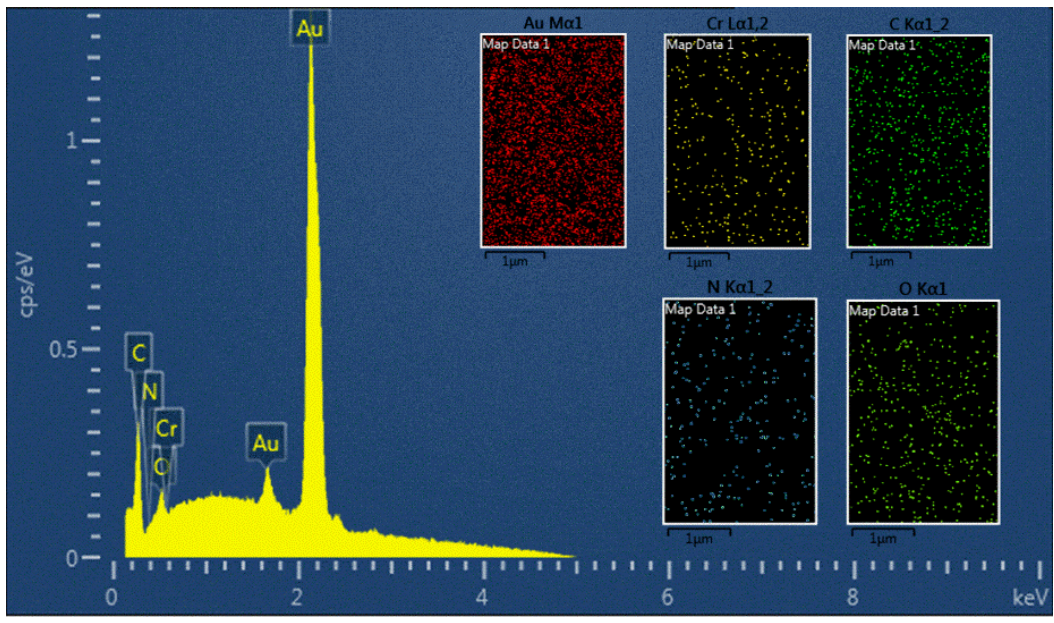

Figure S1. The EDS spectrum of the PU nanospiked gold electrode and EDS mapping of $\mathrm{Au}, \mathrm{Cr}$ $\mathrm{C}, \mathrm{N}$, and $\mathrm{O}$. With accelerating voltage of $5.0 \mathrm{kV}$ and probe current of medium- 13 .

\section{Cyclic Voltammograms in $0.5 \mathrm{M} \mathrm{H}_{2} \mathrm{SO}_{4}$ and Calculation of Electrochemical Active Area}

The PU nanospiked gold electrode was characterized by conducting cyclic voltammogram measurement in $0.5 \mathrm{M} \mathrm{H}_{2} \mathrm{SO}_{4}$ with scan rate of $50 \mathrm{mV} / \mathrm{s}$, scan voltage of $-0.1 \sim 1.5 \mathrm{~V}$. The peak at around $0.92 \mathrm{~V}$ vs. $\mathrm{Ag} / \mathrm{AgCl}$ reference potential is the Au reduction peak ${ }^{1}$. Compared with flat PU electrode, the redox peak of PU nanospiked gold electrode was obviously larger (Figure S2).

The electrochemical active area of the PU nanospiked gold electrode can be determined by integration of the reduction peak of gold oxide located at $+0.92 \mathrm{~V}^{1}$. A value of $400 \mu \mathrm{C} / \mathrm{cm}^{2}$ was used as standard reference charge ${ }^{2}$. Therefore, the electrochemical active areas of the PU nanospiked gold electrode and flat PU gold electrode proposed in this work were calculated to be $0.1323 \mathrm{~cm}^{2}$ and $0.0717 \mathrm{~cm}^{2}$ respectively. 


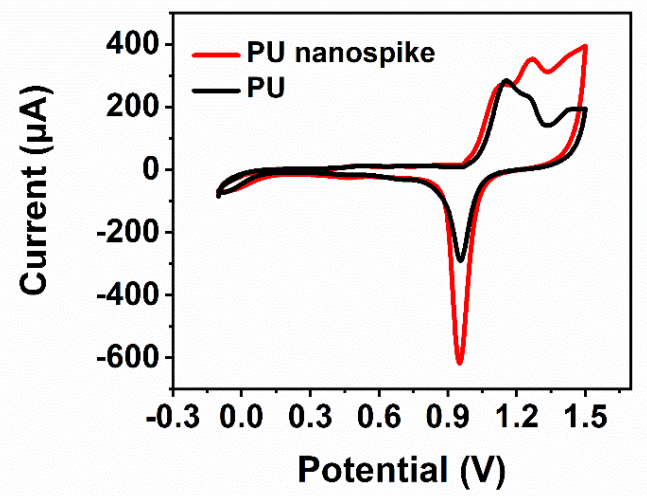

Figure S2. CV of bare PU nanospiked gold electrode in $0.5 \mathrm{M} \mathrm{H}_{2} \mathrm{SO}_{4}$, with scan rate of $50 \mathrm{mV} / \mathrm{s}$.

\section{Stability of the Electrode with Cyclic Voltammetry}

The stability of the electrode was evaluated by cyclic voltammetry with 50 scans in $1 \mathrm{M} \mathrm{KCl}$ containing $5 \mathrm{mM} \mathrm{K}_{3}\left[\mathrm{Fe}(\mathrm{CN})_{6}\right] / \mathrm{K}_{4}\left[\mathrm{Fe}(\mathrm{CN})_{6}\right]$. The results showed that the stability of the PU nanospiked gold electrode was good. It's enough for disposable electrochemical sensor.
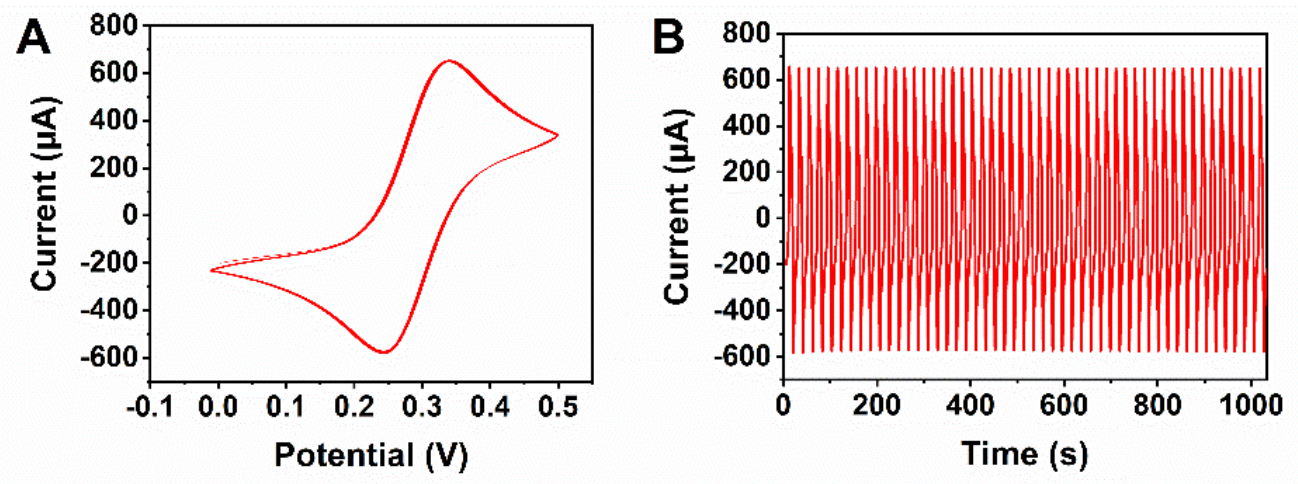

Figure S3. CV (A) and plot of current to time (B) of bare PU nanospiked gold electrode in $1 \mathrm{M}$ $\mathrm{KCl}$ containing $5 \mathrm{mM} \mathrm{K}_{3}\left[\mathrm{Fe}(\mathrm{CN})_{6}\right] / \mathrm{K}_{4}\left[\mathrm{Fe}(\mathrm{CN})_{6}\right]$, with scan rate of $50 \mathrm{mV} / \mathrm{s}, 50$ scans. 


\section{Optimization of Experimental Conditions}

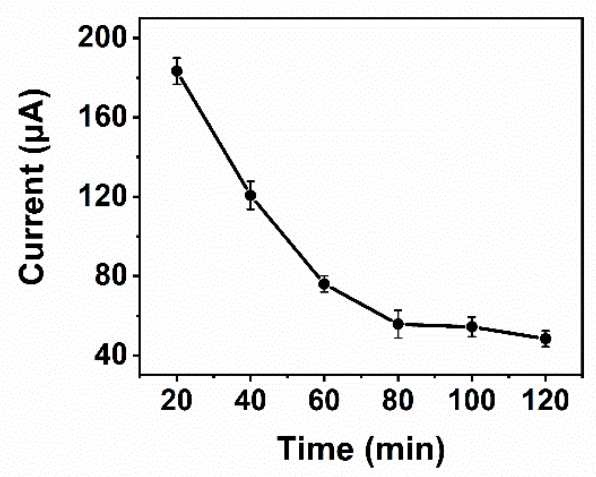

Figure S4. Effects of reaction time on the current responses of the immunosensor toward 100 $100 \mathrm{pg} / \mathrm{mL}$ TcdB.

\section{REFERENCES}

(1) Lai, L. J.; Yang, Y. W.; Lin, Y. K.; Huang, L. L.; Hsieh, Y. H., Surface Characterization of Immunosensor Conjugated with Gold Nanoparticles Based on Cyclic Voltammetry and X-Ray Photoelectron Spectroscopy. Colloid Surf. B-Biointerfaces 2009, 68 (2), 130-135.

(2) Trasatti, S.; Petrii, O., Real Surface Area Measurements in Electrochemistry. Pure Appl. Chem. 1991, 63 (5), 711-734. 